\title{
A Comparative Study of Fingerprint Individuality Models
}

\author{
Oluwatayo Samuel Ogunlana \\ Adekunle Ajasin University, Akungba Akoko, Nigeria \\ Information and Communication Technology Application Centre
}

\begin{abstract}
The use of fingerprint as a means of identification and verification of human identity cannot be over-emphasized in our society due to its reliability, immutability and individuality. It is still the most reliable biometric used to identify individual. Application areas include but not limited to Police, Security, access control and investigation of criminal cases. Several models have been developed for quantitatively established the degree of fingerprint individuality. These models are based on grids, polar systems, fixed probabilities, relative measurements and generative distributions. This paper provides a comparative study of commonly known fingerprint individuality models with emphasis on methodologies, strengths and weakness.
\end{abstract}

\section{General Terms}

Pattern Recognition

\section{Keywords}

Fingerprint, individuality, Probability of random correspondence, minutiae and ridges, model

\section{INTRODUCTION}

Over the years, fingerprints have performed crucial roles in identification and verification of person to ascertain identity. This is possible because fingerprint patterns do not change with time [1-2].
The study of fingerprint individuality started in the late 1800 s [3] and continue till the present day. Fingerprint individuality problem can be formulated based on the probability that any two individuals may have sufficiently similar fingerprints in a given target population. During the past decades, several models have been developed in order to establish the individuality (uniqueness) of two fingerprints. Most of these models which are only based on minutiae property, finds out the probability of false correspondence, which establish the probability of two different individuals being identified as the same based on their fingerprint features $[4,21]$. The broad classification of the fingerprint individuality models based on method of analysis is presented in Figure1, which are categorized into five different categories as follows, gridbased models which contains Galton and Osterburg, polar system model contains Roxburg model, fixed probability models which includes Bose, Henry and Balthazard models, relative measurement models contain Champod model and Trauring model, and generative models which is the newly developed model which its computation is based on probability of random correspondence (PRC) [4]. Each of these models focuses on fingerprint minutiae. Sections 2 and 3 of the paper present some fingerprint individuality models and review of some research works on fingerprint individuality models respectively. The conclusion drawn is also presented in Sections 4.

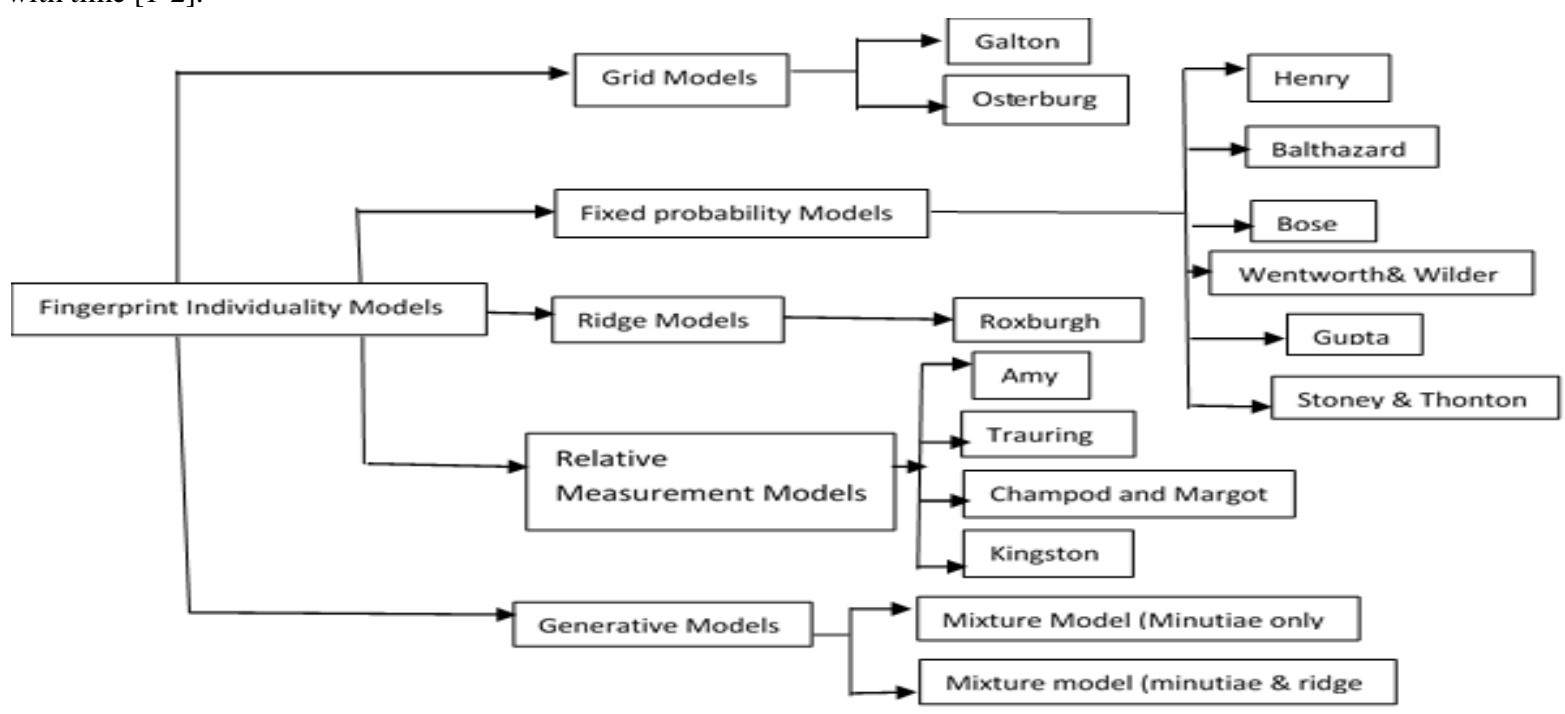

Figure 1: Classification of fingerprint individuality models based on method of analy sis

\section{FINGERPRINT INDIVIDUALITY MODELS}

The summaries of the various fingerprint individuality models are presented below:

\subsection{Grid Models}

The models in this categories use grids to divide fingerprint images into individual square, which are used to examine the distribution of minutiae. Grid models subsequently subdivided into the following models: 


\subsubsection{Galton Model}

Galton [9] made the first attempt to determine the PRC of fingerprint features in 1892. Prior to this work, there was no documented attempt to quantify a probabilistic measure concerning the individuality of fingerprint. The author developed the model by dividing a fingerprint into smaller square regions, such that the ridge detail within each region could be treated as an independent variable [4-6]. Galton focused on a more systematic methodology comprising of marking minutiae location on both fingerprint and attempted to match minutiae location [9]. Galton used a six ridge interval square region and then assigned a probability of one half $(1 / 2)$ for finding the existing minutiae configuration given the surrounding ridges. After critical examination of the minutiae, he observed that the coincidence of these minutiae could serve as evidence of individuality [2]. Given the surrounding ridges, the total area of a complete fingerprint was estimated to consist of 24 such square region. The probability of random correspondence (PRC) of a specific fingerprint configuration is obtained from [4]:

$P(M / N)=\left(\frac{1}{2}\right)^{24}=5.96 \times 10^{-8}$

Where $\mathrm{M}$ denotes specific fingerprint configuration, $\mathrm{N}$ is the surrounding ridges.

Galton further estimated the chance that a particular configuration of surrounding ridges will occur under the following two conditions:

i. the occurrence of general fingerprint pattern type, and,

ii. the occurrence of the accurate number of ridges entering and exiting each of the 24 regions.

Galton therefore predicted the PRC of finding a given fingerprint as follows [4-5]:

$P($ Fingerprint Configuration $)=\frac{1}{16} \times \frac{1}{256} \times\left(\frac{1}{2}\right)^{24}=$

$1.45 \times 10^{-11} \quad$ (2) Where $\frac{1}{16}$ is the probability of

occurrence of a specific fingerprint type such as arch, tented arch, left loop, right loop and whorl, and $\frac{1}{256}$ is the probability of occurrence of the correct number of ridges entering and exiting each of the 24 region.

Galton's methodology for assessing the individuality of minutiae events is largely subjective and ad-hoc, since it is purely relied on Galton's own analytical skills and experience, while lacking statistical reasoning. Also, the addition of value $\frac{1}{256}$ is arbitrary and lacking empirical foundation, while the pattern probability value used is a coarse approximation of pattern type occurrence. Lastly, the minutiae types were also assumed to be of equal probability, while no attempt was made to incorporate minutiae directional information [5].

However, Galton's model was criticized by Pearson [4]. His criticism was centered on Galton's assumption that there is a probability of $1 / 2$ for the occurrence of any particular ridge configuration in one of the six ridge integral regions of the surrounding ridges. Pearson considered this assumption drastic and suggested an alternate approach for determining the probability of a particular configuration. He argued that there might be $6 \times 6$ possible minutiae locations within one of Galton's six-ridge-square regions. Pearson therefore computed the probability of any given configuration using the equation:

$$
\begin{aligned}
& P(\text { Fingerprint Configuration }) \\
& \qquad=\frac{1}{16} \times \frac{1}{256} \times\left(\frac{1}{36}\right)^{24}
\end{aligned}
$$

$$
=1.09 \times 10^{-41}
$$

Pearson observed that the actual probability would be smaller based on the following two reasons

i. The minutiae are not uniformly restricted to a single minutia in each Galton region.

ii. The variability in minutiae type.

\subsubsection{Osterburg Model}

This model classifies fingerprints into loops, arches and whorls, which are further divided into subclasses. Osterburg made the following assumptions in his individuality model [56,10]:

i. A fingerprint is a combination of grids

ii. There are 13 possibilities in each cell, which can be 10 minutiae, broken ridge, empty cell or any other multiple occurrences of minutiae.

iii. The cells are statistically independent.

Osterburg model extended the identification using minutiae which involves the use of some minutiae types to characterize a fingerprint [5]. Osterburg model divided fingerprints into discrete cells with the size $1 \mathrm{~mm} \times 1 \mathrm{~mm}$ grid. He computed the frequencies of 13 types of minutiae features from 39 fingerprints and estimated that 12 ridge endings will match between two fingerprints based on an average fingerprint area of $72 \mathrm{~mm}^{2}$ with the PRC of $1.25 \times 10^{-20}$ [4]. Osterburg also computed the probability of a particular configuration as a multinomial distribution as follows: if $p_{0}$ is the probability of empty cells, $t_{0}$ is the number of empty cells, $p_{1}$ is the probability of ending ridges, $t_{1}$ is the number of ending ridges, $p_{12}$ is the probability of multiple occurrences, $t_{12}$ is the number of multiple occurrences, then the PRC of a given configuration is express as follows [5]:

$$
P R C=p_{0}^{t_{0}} p_{1}^{t_{1}} \ldots p_{12}^{t_{12}}
$$

The sum of the weights parameters assigned to each minutiae type is the negative log probability for the minutiae and expressed as follows [5]:

$$
E=-\sum_{i=0}^{12} t_{i} \log _{10} p_{i}
$$

Where $\mathrm{i}$ is the number of weight parameters.

Experts observed that the minimum number of minutiae required to identify a fingerprint is twelve. Osterburg conducted experiment to determine the robustness of the model formulated with varying cell sizes. It was found that there is significant change in the probability of random correspondence. If there is an arbitrary cell size that approximates the independence relation well, then the model with cell size 1 is accurate because the probabilities do not vary with cell size [10]. The limitations of the Osterburg model include the assumption that minutiae type frequencies are independent of specific spatial regions within the fingerprint. In addition, there is no attempt to consider minutiae orientation in the PRC calculations, while the dataset used to find such cell configuration frequencies was small due to non-availability of computational tools. The work of authors in [18] was an enhancement to the Osterburg model. The authors developed a modified grid method for comparison of fingerprints. The existing classical method of fingerprint comparison with lens to locate minutiae in direction is time consuming cumbersome. The earlier Osterburg grid was segmented with equal size squared and 
each grid occupying an identical position on each fingerprint, which made the model difficult and impracticable because of the size of each block in the grid was $1 \mathrm{~mm}$, as such, reduces the visibility of fingerprint minutiae. The authors developed a modified grid by drawing circular shape with a different of 10 degree covering 0-360 degree angle. The circle further segmented with cubes of equal dimensions can either be used manually or digitally for comparison of fingerprints. The center of the grid is placed on the fingerprint core location and the cubes are utilized to find ridge densities. The ridge orientation of core point was computed and the result of this model is superior to the earlier Osterburg model. The modified grid enables the expert to perform one-to-one comparison of fingerprint. However, there is systemic collapse with bad and porous images.

\subsection{Fixed Probability Models}

\subsubsection{The Henry Model}

This is the first model used by Henry in 1900 to consider minutiae as independent variable events along with core/delta, ridge count and classification weight. This model employs a fixed PRC for the occurrence of one minutia as an approach to the fingerprint individuality model. The model calculates the probability of a particular configuration of $\mathrm{N}$ minutiae using the equation:

$$
P R C=(\beta)^{N}
$$

Where $\beta$ is the fixed probability

Henry chose an arbitrary probability of $1 / 4$ for the occurrence of each minutia (ridge ending and bifurcation), pattern type, and the core/delta ridge count. The author made an extensive study of occurrence of fingerprints and indexed them into 8 classes which comprises of arch, tented arch, left loop, right loop, whorl, central-pocket whorl, double-loop whorl and accidental whorl. The four different whorl classes were latter combined into one class whorl. The Henry system with five classes latter reduced to four classes by combining the plain arch and tented arch classes to form the arch class. These five classes differ in terms of the global flow patterns of the ridge curve. The problems with the Henry model are non-uniform classification proportions, and there is misclassification of some fingerprint images into different Henry classes. [17]. Also, there is no attempt to empirically quantify the values suggested in the model, which made these values to be largely arbitrary. The is no relevant empirical evidence backed the decision on the selected probability values.

\subsubsection{Balthazard Model (1911)}

Balthazard was the first to provide a numerical standard in its probabilistic model. He suggested that a minimum of 17 corresponding minutiae would be needed to identify an individual with certainty. Balthazard later suggested the number could be reduced depend on the number of fingerprint available. He also assumed that for each minutia either ridge ending or ridge bifurcation, there are four possible events [4]:

i. Bifurcation directed to the right

ii. Bifurcation directed to the left

iii. Ending directed to the right

iv. Ending directed to the left

$\mathrm{He}$ also assigned fixed probability as $1 / 4$ for these events and concluded that to observe $\mathrm{N}$ coincidentally corresponding minutiae, it would be necessary to examine $4^{\mathrm{N}}$ fingerprints.

\subsubsection{Bose Model}

This model [3-4] assumed the probability of a minutia occurring to be $1 / 4$ based on the four possibilities at each square ridge interval location namely, dot, ridge bifurcation, ridge ending and continuous ridge [4]. Bose's model does not consider the possible events for each minutia, but rather consider possible events at each ridge-interval location.

\subsubsection{Wentworth and Wilder Model}

This model [4] considered four types of minutiae, namely, ridge ending, bifurcation, islands and breaks. This model assumed the probability of a minutia occurring to be $1 / 50$ thinking that $1 / 4$ was too high for the probability of occurrence.

\subsubsection{Stoney and Thornton model}

The model was developed to study the probabilities of random correspondence of various types of minutiae, their orientations, number of neighboring minutiae, and distance/ridge counts to the neighboring minutiae. The model calculated the probability of a minutiae configuration by conjoining the probabilities of individual events in the configuration, and also proposed a linear ordering of minutiae and recursively estimated the probability of $n$-minutiae configuration from the probability of an (n-1)-minutiae configuration and the occurrence of a new minutiae of certain type/orientation of a particular distance/ridge counts from its nearest minutiae with the (n-1) minutiae configuration [6]. The model computed the probability of false association by accounting for the various possible linear orderings which could initiate the search for correspondence. The weakness of Stoney and Thornton model include the consideration of variation among fingerprints from the same source, was not sufficiently addressed. Also, their tolerances for minutiae position were derived from successive fingerprint images under normal conditions and are far too low to be applicable in actual fingerprint comparisons. The assumption of independent between orientation and location, inaccurate and over-conservative orientation probability calculations and statistics are mainly centered on the minutiae above the core location [5].

\subsection{Polar System Models \\ 2.3.1 Roxburgh Model}

This model involves the following features [11]:

i. The use of a polar coordinate system to uniquely identify minutiae

ii. Treatment of correlation among neighboring minutiae

iii. Adjustments for fingerprint quality and for connective ambiguity

iv. Consideration of variation in the position of the minutiae configuration relative to the pattern core.

Roxburgh proposed more comprehensive analysis to compute the probability of a fingerprint configuration [4]. His analysis was based on considering a fingerprint as a pattern with concentric circles with one ridge interval from the core, in a polar coordinate system as shown in Figure 2. 


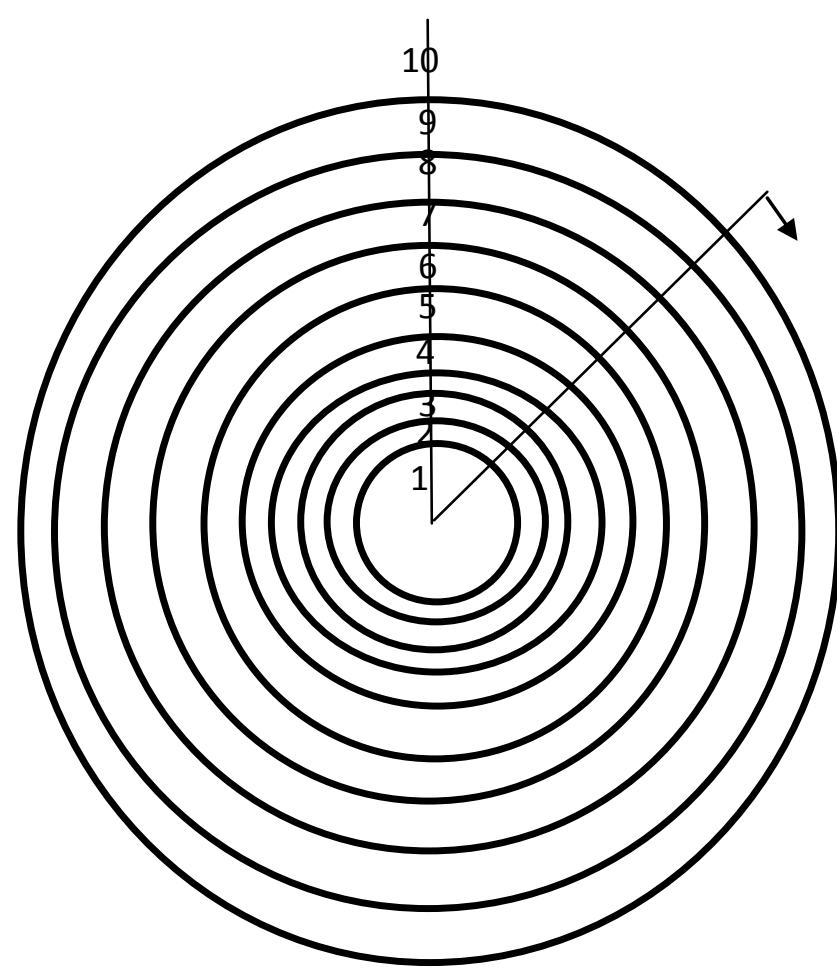

Figure 2: Concentric circle structure with origin at the core

An axis is drawn extending upward from the origin and is rotated in a clockwise from the vertical axis. Fingerprint minutiae position and orientation, ridge count (concentric circle number) as well as minutiae types for each minutia, are recorded [6].

Roxburgh also incorporated a quality measure of the fingerprint into his computation and assumed that the ridge count and minutiae types were independent. He computed the probability of a particular fingerprint configuration using:

$$
P(\text { Fingerprint Configuration })=(N / P) \times(D / Y Z)^{K}
$$

Where $\mathrm{P}$ is the probability of encountering a particular fingerprint type and core type, D is a measure of quality ( $D=1.5$ for an average quality fingerprint and $D=3.0$ for a poor quality fingerprint), $\mathrm{Y}$ is the number of semicircular ridges in a fingerprint, $\mathrm{Z}$ is the minutiae types and $\mathrm{K}$ is number of minutiae. The major weakness of Roxburgh model is that the model did not incorporate minutia resolution into the model which is a fundamental aspect of fingerprint individuality. Also, the arbitrary quality weights used which are assigned subjectively, and uniform density of minutiae among all regions of the fingerprint. In addition, the acknowledgement of more than one possible core location to be the center of the circle configuration creates more than one possible PRC calculation, as the values may differ depending on the circle configuration alignment.

The author in [30] proposed a fingerprint matching algorithm using the combination of both the ridge features and the minutiae features. The author incorporated ridge features such as ridge count, ridge length, ridge curvature direction and ridge type. The minutiae feature used are minutiae types, orientation and position. The ridge features are extracted by defining the ridge-based coordinate system in a skeletonized image form. The result of the proposed method yielded a higher matching scores compared to the convectional minutiae based alone. However, the algorithm failed with low quality images and preprocessing method id required in order to reduce enhancement errors.

A fingerprint matching algorithm which establishes both the ridge and minutiae correspondences between two fingerprints is presented by the authors in [20]. For each of the minutiae pair, the correspondence ridge matching process is performed which incrementally matches the remaining minutiae and ridges and the matching scores was computed for both minutiae and ridges. An effective fingerprint matching algorithm that matches both the minutiae and ridges was developed. The algorithm developed was able to handle the non-linear deformation and poor quality fingerprint images. However, the alignment algorithm performed poorly for fingerprint with fewer minutiae.

\subsection{Relative Measurement Models}

\subsubsection{Amy model}

This model [4-6] defined two general contributions to fingerprint individuality. They are, variability in minutiae type and variability in number and position of minutiae. Amy proposed a probability measure for a given minutiae type and minutiae position, that was utilized to help derived a PRC value. Minutiae could either be bifurcation or ridge ending. $\mathrm{He}$ used a database of 100 fingerprints for experimental evaluation, Amy calculated relative frequencies rather than assuming equal probabilities for each type of minutiae. The relative frequency results were 0.40 and 0.60 for bifurcations and ridge endings respectively. While the probabilities of $3 / 4$ and $1 / 4$ were used for the two relative opposing directions [29]. Amy also computed variations in the number and spatial position of minutiae. Given a square region of ridges with $r$ ridges interval on the size, the probability of a specific number and spatial location of a minutiae, $\mathrm{M}$ existing in a square is given as follows:

$P(M)=\frac{P(r, x) \cdot N(r, t)}{N(r)}$

Where $P(r, x)$ is the probability that $x$ minutiae exist within the square, $N(r)$ is the total number of arrangements of the $x$ minutiae, $N(r, t)$ is the total number of arrangement in a given configuration of $x$ minutiae.

Some of the limitations of this model include the assumption of uniform minutiae density and minutiae location-type independence. Also, the relative positioning proposed does not account for precise location of minutiae occurring at a given ridge. Hence, the estimate for the number of configurations of minutiae within a given square region may not be entirely accurate [5].

\subsubsection{Trauring Model}

Trauring $[14,27]$ was the first to concentrate explicitly on measuring the amount of details needed to establish a correspondence between two fingerprints from the same finger (intra class variation) using a system of fingerprint identification, and observing that corresponding fingerprint features in impressions of the same finger could be displaced from each other by as much as 1.5 times the inter ridge distance [3]. Trauring further made the following assumptions:

i. Minutiae are randomly distributed.

ii. There are two minutiae types, bifurcation and ridges ending iii. The two minutiae types are equally likely to occur 
iv The two possible orientations of minutiae are equally likely to occur

v. Minutiae type, orientation and position are independent variables.

Trauring computed the probability of false identification of fingerprint as follows [3]:

$P=10 \mu .9 \pi \frac{\varphi}{16}^{i}$

Where $i$ is number of test minutiae, $\varphi$ is the minutia density (minutia per square mean pattern wavelength), $\mu$ is the probability of matching reference minutiae in the false fingerprint.

The most important feature of Trauring's model is his concept of reference minutiae. He used the locations of three reference minutiae to determine the individuality of fingerprint. Positions of the remaining test minutiae were determined relatively to these reference minutiae. Although no other fingerprint model explicitly distinguishes between reference and test minutiae. However, the concept of test and reference minutiae remain undeveloped in its application to conversional fingerprint comparison. Although, an innovation modelling association was used with regards to the proposed theoretical AFIS system, the model is novel in assumptions and considerations. Incorrect assumptions of uniform minutiae density and type frequencies were used. In addition, estimated values for the parameters used are lacking a strong empirical foundation as they rely on a very small sample set.

\subsubsection{Kingston model:}

Kingston proposed a model for fingerprint individuality by dividing his model into three probability calculations for a given configuration of minutiae. He first calculated the probability of finding the observed size, next, he calculated the probability that the particular minutiae positions would be observed. Finally, he calculated the probability that minutiae of the observed type would occupy the positions. The Poisson distribution was employed to measure minutiae density, assuming the uniform density for all fingerprint regions and classes. The probability of the observed minutiae $(\mathrm{N})$ in a given fingerprint region is computed as follows:

$\mathrm{P}(\mathrm{N}-$ minutiae $)=\left(e^{-y}\right)\left(y^{N} / N !\right)$

Where $\mathrm{N}$ is number of minutiae and $\mathrm{y}$ is expected minutiae number. The minutiae density was measured for the specific type of finger pattern and location.

Kingston next computed a probability for the observed positioning of minutiae. He assumed that each minutia occupied a square region of $0.286 \mathrm{~mm}^{2}$. Each square containing a minutia was assumed to exclude other minutiae existing within the square. Thus, a square region could be extended to a size of $0.571 \mathrm{~mm}^{2}$, being the largest possible square with only one minutia. Under this assumption, Kingston proceeded as follows: consider $\mathrm{N}$-minutiae occurring in a region $\mathrm{S}$ square millimeters in area, the number of minutiae position within this region is computed as follows:

$$
\mathrm{N}=(S) /(0.571)^{2}=(S) /(0.082)
$$

Where $\mathrm{N}$ represents number of minutiae

Kingston also estimated the probability of correspondence in minutiae types, by determining the relative frequencies of minutiae types. The probability for a correspondence in minutiae type was calculated as the product of the relative frequencies for each of the $\mathrm{n}$ minutiae.

The general similarity between the Kingston model and the Amy model are

i. $\quad$ Both models determine the probability of a particular number of minutiae in a region of a given size.

ii. They both compute the possible permutations of minutiae positions.

iii. They consider variation in minutiae type.

The principle difference between the two models is that, Amy attempts to describe minutiae position within the ridge structure, whereas Kingston ignores this structure and uses the coordinates of minutiae positions. The limitations of the model include the assumption of minutiae type probability being independent of its spatial region. In addition, positioning calculations assume uniform density and the minutiae spatial information for a given configuration of minutiae is not used. Also, no orientations of minutiae were considered in the probability calculations.

\subsubsection{Champod and Margot Model}

This model is specifically designed to search for minutiae in a fingerprint by use of image processing algorithm to aid the detection of minutiae features [9]. The algorithm reduced the fingerprint images into a skeleton image to ensure the accuracy of minutiae position and orientation when compared with the original images [6]. The model incorporated ridge ending and bifurcation as major minutiae features used. The positions of the minutiae are calculated relative to the core point. Orientations were defined relative to other minutiae when the ridge flow was in a constant direction which is measured relatively to the vertical axis and the length of minutiae were measured for experimental purposes. The authors observed that, density of minutiae was highly concentrated around singular points (core and delta) in comparison to other areas of the fingerprint. The number of minutiae was seen to follow a Poisson distribution in the area above the core, but the region below the core had minor deviations from the distribution [5].

\subsection{Generative Models}

These models incorporated $10 \mathrm{th}$ the location and orientation of extracted minutiae into a mixture model. The authors in [4] use mixture model using Hyper geometric and Binomial distributions for minutiae. The following conditions were assumed by the authors in their fingerprint individuality model [4-5]:

(i). Only two types of minutiae are considered, ridge ending and bifurcation.

(ii). Minutiae are uniformly distributed with the restriction that they are not very close to each other.

(iii). Correspondence between minutiae in template and input fingerprints is independent and have an equal weight.

(iv). The fingerprint image quality is not taken into account since it is very difficult to assign reliably a quality index to a fingerprint.

(v). Ridge widths are assumed to be same.

(vi). There exists only one correct alignment between fingerprints.

The input and the template minutiae sets I and T, respectively are computed as follows [4]:

$$
I=\left\{\left\{x_{1}^{\prime}, y_{1}^{\prime}, \theta_{1}^{\prime}\right\},\left\{x_{2}^{\prime}, y_{2}^{\prime}, \theta_{2}^{\prime}\right\}, \ldots,\left\{x_{n}^{\prime}, y_{n}^{\prime},\right.\right.
$$




$$
T=\left\{\left\{x_{1}, y_{1}, \theta_{1}\right\},\left\{x_{2}, y_{2}, \theta_{2}\right\}, \ldots,\left\{x_{m}, y_{m}\right.\right.
$$

Where $(x, y)$ and $\left(x^{\prime}, y^{\prime}\right)$ are locations of input and the template minutiae respectively, $\theta$ is the angle of the ridge. A minutiae $j$ in the input fingerprint is corresponding or matching to the minutiae $i$ in the template, if and only if

$$
\begin{aligned}
& \sqrt{\left(x_{i}^{\prime}-x_{j}\right)^{2}+\left(y_{i}^{\prime}-y_{j}\right)^{2}} \leq \mathrm{r}_{0} \\
& \operatorname{Min}\left(\left|\theta_{i}^{\prime}-\theta_{j}, 360-\right| \theta_{i}^{\prime}-\theta_{j} \mid\right) \leq \theta
\end{aligned}
$$

Where $r_{0}$ is the tolerance in the distance and $\theta_{0}$ is the tolerance in angle.

The authors in [7] involved a Gaussian mixture model and Von-mises Distributions to represent minutiae location and direction. This model is flexible in such that it can represent the observed distributions through other fingerprint database. The model also assumes non-independence of minutia location and orientations.

The minutiae location and direction are modeled as follows: let $\mathrm{X}$ denotes a generic random minutiae location and $\mathrm{D}$ denote its corresponding direction. The minutiae location is distributed according to the density as expressed using:

$f_{g}^{X}\left(s \mid \mu_{g}, \sum_{g}\right)=\emptyset_{2}\left(s \mid \mu_{g}, \sum_{g}\right)$

Where $\emptyset_{2}$ is the bivariate Gaussian density with mean $\mu_{g}$ and covariance matrix $\sum_{g}$.

The minutiae direction (O) is modeled using a Von-Mises distribution using the equation:

$f_{g}^{D}\left(\theta \mid v_{g}, k_{g}, p_{g}\right)=p_{g} v(\theta) . I\{0 \leq \theta<\pi\}+$

$\left(1-p_{g}\right) v(\theta-\pi) . I\{\pi \leq \theta<2 \pi\}$

Where $I\{A\}$ is the indicator function of the set $\mathrm{A}$ (that is $I\{A\}=1$ if $\mathrm{A}$ is true and 0 otherwise), $p_{g}$ is a real number between 0 and 1 , and $v(\theta)$ is the Von-Mises distribution modelling the angular random variables in $[0, \pi]$ with $v_{g}, k_{g}$ are the Von-mises parameters.

The generative model for minutiae and ridges was demonstrated by authors in [15]. The forth parameter ridge type was added to the three existing parameters of $x, y, \theta$ of the extracted minutiae and a generative model is built for these parameters as follows:

$f\left(s, \theta, \tau \mid v_{G}\right)=\sum_{g}^{G} \tau_{g} f_{g}^{X}\left(s \mid \mu_{g}, \sum_{g}\right)$.

$f_{g}^{D}\left(\theta \mid v_{g}, k_{g}, p_{g}\right) \cdot f_{g}^{T}(\tau)$

Where $f_{g}^{T}(\tau)$ is an empirical distribution of the frequencies of ridge types.

\subsection{Comparison of Different Fingerprint Individuality Models}

The comparison of various fingerprint individuality models based on their configuration, the computed PRC values and types of minutiae used. Basically, it was observed that different models make use of difference minutiae features and follow different methods in computing their PRC. Many of the earlier models were mere guesses of probability values and were very primitive. Majority of these models considered ridge endings and ridge bifurcations while others co-opted other minutiae features so as to facilitate the models to get accurate and a much lower PRC [5]. Models also differ in the number of fingerprints used to compute their PRC. The earlier models take a few fingerprints to represent the general population into consideration due to the manual computation of PRC involved, while the recent models considered larger fingerprints for the representation of the general population. Earlier models like Galton's divides fingerprint into independent grids and find the PRC as a product of the probability of individual grids, others use a relative measure between minutiae for better matching [6]. The newer models are the generative models which are based on distribution of minutiae of a database and then find the PRC by generating fingerprint from the model.

\section{SYNOPSIS OF SOME RESEARCH WORKS ON FINGERPRINT INDIVIDUALITY MODELS}

The summary of the objectives, methodologies and the limitations of some research works that are based on the models presented in the preceding Sections is presented in this Section.

The authors in [4] proposed a fingerprint individuality model that relied on fingerprint image features. The authors extracted the minutiae location and direction to compute the probability of false random correspondence between two fingerprints minutiae. The extracted minutiae were used for matching by validate the minutiae occurrences, matching probabilities and probability of random correspondence (PRC) with benchmarked fingerprint images. The distributions from the theoretical model did not fit the empirical distribution. The probability for larger numbers of impostor minutiae pairing per impostor comparison were understated by the theoretical

distributions. Also, since minutiae feature extraction was fully automated, spurious minutiae may exist in some of the recorded minutiae pairings resulting in an inflated number of impostor minutiae pairings. The authors in [7] presented statistical methods for assessing the individuality of fingerprint. The extracted minutiae location was modelled using bivariate Gaussian density while minutiae direction was modelled using a Von-Misses distribution method. In order to compute the probability of random correspondence, a Poisson distribution with mean parameters derived from the fitted mixture distribution was used. The empirical probabilities of matching minutiae pair were computed and compared with the theoretical probability. The model did not consider singular points features. Also, the algorithm is computationally bulky and time complexity.

Minutiae-based fingerprint individuality model was presented in [10]. Complete spatial randomness (CSR) technique was used to compute the Euclidean distance between extracted minutiae. The minutiae positions of each fingerprint were manually marked by experts and only minutiae locations were considered for the matching. An experiment was performed on selected

fingerprints from three fingerprint databases where the empirical impostor distribution was compared to the simulated distribution of the proposed model, the experimental results indicated that the simulated distribution of the proposed method was much closed to the observed 
empirical distributions. The model requires large sample size of database for categorization with respect to quality and number of fingerprints and does not consider minutiae orientation for the matching. Also, the model failed to detect spurious points. Although, the authors do not assume a uniform distribution for the directional difference of spatially minutiae, their assumptions of a homogeneous minutiae spatial distribution strongly against empirical observations, ultimately leading to inaccuracy PRC estimates.

The authors in [11] proposed a generative model for fingerprint individuality using ridge types. The authors observed only minutiae location and direction were used to determine the probability of random correspondence (PRC) between two fingerprints, not much emphasis was given to generative models using ridge points for fingerprint matching. The authors incorporated ridge information into the generative model by using a third distribution for ridge types. The joint probability of minutiae location, orientation and ridge type is modeled as a mixture distribution. Though the developed model offered a more accurate fingerprint representation model for PRC computation, the performance of the model is diminished with low quality images. Also, only macro scale spatial characteristics are discovered, while any statistical spatial relationships within clusters (between neighboring minutiae) are ignored. The authors in [12] proposed a generative model for fingerprint minutiae by investigating the correlation between the ridge orientation field and extracted minutiae. The model used a large training data size. The authors implemented the model using both the extracted features manually marked by experts and the features extracted by automated matchers. The proposed model performed better with small number of training fingerprints and performed badly with the minutiae extracted in singularity area (areas surrounded by core/delta) which later displayed low reliability. A statistical modeling of fingerprint individuality was proposed in [13]. The authors used spatial point pattern analysis to extract the minutiae location, direction and type and formed two dimensional point patterns. Ripley's K function was used as statistical property for comparison. The minutiae patterns were marked by human experts and the missing minutiae during marking were approximated using Bernoulli process. The model can serve as a foundation for more accurate theoretical analysis of the fingerprint individuality. The model accurately represents small scale over-dispersion and large scale clustering of a given population. Also, the simulating realization of the model can be used in fingerprint synthesis systems for generating more realistic artificial fingerprints. However, the authors make an unfounded assumption that every fingerprint has similar clustering and over-dispersion characteristics. The $\mathrm{K}$ function of the pair potential Markov process cannot be express in a closed form. This will greatly jeopardize the usability of the proposed model for any further theoretical analysis.

An individuality model for online signature using Fourier domain representation of a fingerprint signature was proposed in [14]. The model is hinged on the extraction of the coordinates of the sample points. During enrolment, the user supplies a number of reference signature which are used to measure the variation of the query signature. The range of each harmonic is divided into a constant number of bins centered on the mean of that feature for the reference signature. During marching, query signature is said to match the reference set if its descriptor fall in same bin as the mean of the reference set of the harmonic.
An algorithm for assessing fingerprint individuality in presence of noisy minutiae was presented in [16]. The authors modeled the minutiae location and direction using Bivariate Gaussian density and Von-Mises distribution respectively. The salient features of minutiae occurrence were modeled using clustering tendencies and spatial dependence between the location and orientation of the minutiae. The PRC was then computed as a measure of fingerprint individuality. PRC value focuses only on assessing the probability of corresponding evidential features from fingermarks of different fingers. Thus, PRC model lacks the important evidential consideration of within-finger feature variability.

A generalized mixed model framework for assessing fingerprint individuality in presence of varying image quality was proposed in [17]. The PRC was computed for location and direction of a pair of fingerprint image using Euclidean and angular distances respectively. The model addressed the problem of noise as a result of low quality images. However, the model for minutiae occurrences and matching are not validated based on actual fingerprint images. The authors in [22] presented a fingerprint individuality model with pattern, ridge and pore features. The authors modelled the extracted minutiae position and direction, computed the mixture density of minutiae, ridge and pore features as well as the probability of random correspondence (PRC) using the Poisson probability mass function. The model required high resolution (about $1200 \mathrm{dpi}$ ) to extract pore features. Also, the theoretical and empirical estimates were affected by factors such as image quality as well as feature extraction and matching algorithms.

\section{CONCLUSION}

Findings from the survey of some of existing models for fingerprint individuality have been presented with emphasis on strengths, weakness, opportunities and threats. The models include the following, grid-based, fixed probability, ridgebased, relative measurement and generative. These models are categorized based on their approaches for measuring individuality. Synopsis of some recent research works on fingerprint individuality management that are premised on these models is also presented with focus on the motivations, objectives, methodologies and the attendant limitations. Future research therefore aims at experimental study of these models and proposing model that will address existing and envisaged common problems of fingerprint-based individuality management as well as some of the limitations of the reviewed works.

\section{REFERENCES}

[1] Iwasokun, G. B. and Akinyokun, O. C. 2014 Fingerprint Singular Point Detection Based on Modified Poincare Index Method, International Journal of Signal Processing, Image Processing and Pattern Recognition, 7(5), pp 259-272

[2] Ogunlana, S.O., Olabode, O, and Iwasokun, G.B. Fingerprint Individuality Model based on Pattern Type and Singular Point Attribute. International Journal of Information Security Science. Accepted for Publication.

[3] Srihari, S.N. and Srinivasan, H. 2007. Individuality of Fingerprints: Comparison of Models and Measurements. Preprint submitted to Elsevier, pp.1-26.

[4] Pankanti, S., Prabhakar, S. and Jain, A.K. 2002. On the Individuality of Fingerprints, IEEE Transactions on Pattern Analysis and Machine Intelligence, 24(8), pp. 1010-1025 
[5] Abraham, J. 2017 Statistical models for the support of forensic identifications. PhD thesis to Centre for Forensic Science, University of Technology, Sydney.

[6] Stoney, D. 2011 Measurement of fingerprint individuality. In Advances in Fingerprint Technology, $2^{\text {nd }}$ Edition.

[7] Zhu, Y., Dass, S. and Jain, A.K. 2007 Statistical Models for Assessing the Individuality of Fingerprints, IEEE Trans on Information Forensics and Security, Vol.2, pp.391-401.

[8] Henry, E. 2004 Classification and uses of fingerprints. Routledge \& Sons London, pp 1-64. Digital edition prepared for http://galton.org by Gava Tredoux.

[9] Champod, C. and Margot, P. 1996 Computer assisted analysis of minutiae occurrences on fingerprints. Proc. International symposium on Fingerprint Detection and Identification.

[10] Chen, J. and Moon, Y. 2007 A Minutiae- based Fingerprint Individuality Model, Proceedings of Computer Society International Conference on Computer Vision and Pattern Recognition (CVPR 2007), 18-23 Minneapolis, Minnesota, USA

[11] Fang, G., Srihari, S.N. and Srinivasan, H. 2007 Generative models for fingerprint individuality using ridge types. IEEE, $3^{\text {rd }}$ International Symposium on Information assurance and Safety.

[12] Zhao, Q., Zhang, Y., Jain, A.K., Paulter, N. G. and Taylor, M. 2013 A generative Model for Fingerprint Minutiae. IEEE.

[13] Chen, J. and Moon, Y. 2008 The Statistical Modeling of Fingerprint Minutiae Distribution with implications for fingerprint individuality Studies. IEEE, 978-1-42442243.
[14] Kholmatov, A. and Yanikoglu, B. 2008 An Individuality Model for online Signature using global Fourier Descriptors. Proc of SPIE.

[15] Su, S. and Srihari, S.N. 2008 Generative Models for Fingerprint Individuality using Ridge Models, IEEE, pp.1-4

[16] Dass, S.C. 2010 Assessing Fingerprint Individuality in Presence of Noisy Minutiae, Department of statistics and Probability Technical Report. Michigan State University.

[17] Dass, S. C., Lim, C. Y. and Maiti, T. 2014 A generalized mixed Model Framework for Assessing Fingerprint Individuality in Presence of Varying Image Quality, Annal of Applied Statistics, 8(3), pp.1314-1340.

[18] Thakar, M. K. and Sharma, T. 2016 Digital grid method for fingerprint identification and objective report writing. Egyptian Journal of Forensic Sciences, pp 194-201.

[19] Choi, H., Choi, K. and Kim, J. 2011 Fingerprint Matching incorporating ridge features with minutiae. IEEE, Transactions of Information Forensics and Security, 6(2), pp 339-345

[20] Feng, J., Ouyang, Z. and Cai, A. 2006 Fingerprint Matching using Ridges. The Journal of the Pattern Recognition Society, pp 2131-2140.

[21] Dass, S.C. 2014 Individuality of Fingerprint: A Review. Springer Verlag London. 2014, pp 1-19.

[22] Chen, Y. and Jain, A. K. 2009 Beyond Minutiae: A Fingerprint Individuality Model with Pattern, Ridge and Pore Features, Proceedings of International Conference on Biometric, pp.523 - 533,Available: http://biometrics.cse.msu.edu/Publications/Fingerprint/C henJainIndividuality_ICB09.pdf 